\title{
Apoptotic Pathway-targeting Antineoplastic Agent
}

National Cancer Institute

\section{Source}

National Cancer Institute. Apoptotic Pathway-targeting Antineoplastic Agent. NCI

Thesaurus. Code C129839.

Any ag ent that directly targets the apoptotic pathway and has an antineoplastic effect. 